\title{
OPTIMAL BEAM OPTICS IN THE TTF-FEL BUNCH COMPRESSION SECTIONS: MINIMIZING THE EMITTANCE GROWTH
}

\author{
M. Dohlus, A. Kabel* ${ }^{*}$ T. Limberg \\ Deutsches Elektronen-Synchrotron DESY \\ Notkestr. 85, D-22607 Hamburg, Germany
}

\begin{abstract}
In the bunch compressing sections of the Tesla Test Facility Free Electron Laser, short bunches travel on trajectories with small bending radii. Thus, coherent synchrotron radiation (CSR) will play a significant role in beam dynamics. The energy loss of the bunch will vary longitudinally as well as transversally across the bunch and will induce an emittance growth. This emittance growth will affect the projective as well as the slice emittance (i.e., the emittance of sub-ensembles of particles with equal longitudinal position). The computer code $\mathrm{TraFiC}^{4}$ is a significantly augmented version of WAKE, which calculates these effects from first principles. One result of simulations is the observation that the slice emittance growth depends crucially on the initial Twiss parameters chosen for the beam. We discuss the mechanisms leading to this dependence. Simulation results for a range of optics will be given.
\end{abstract}

\section{INTRODUCTION}

The Tesla Test Facility (TTF) aims to conduct a proofof-principle experiment for a self-amplified spontaneous emission free electron laser. (SASE FEL). For FEL operation, high peak currents and low transversal emittances are crucial. To reach these quality conditions, the bunch has to be compressed longitudinally after it leaves the gun. In the case of the TTF FEL experiment, this is done in magnetic chicanes. Bunch Compressor II, which has been commissioned recently [1], is the only chicane to be used for the proof-of-principle experiment.

All subsequent calculation are for the BC II design values; i. e. $E=140 \mathrm{MeV}, Q=10^{-9} \mathrm{As}, \sigma_{l, \text { initial }}=1 \mathrm{~mm}$, $\sigma_{l, \text { final }}=250 \mu \mathrm{m}$. The initial emittance used is $\epsilon_{N, i}=$ $0.85 \cdot 10^{-6} \mathrm{~m}$, in accordance with simulations ${ }^{1}$ for the FEL Gun emittance.

A further stage of FEL operation, using smaller wavelengths, higher energy and a longer undulator, requires another stage of compression (Bunch Compressor III) [2]. The low-energy stage Bunch Compressor I will not be used for FEL operation.

When a short charged bunch travels on a bent trajectory, coherent interaction of the bunch's tail with its head (as opposed to usual wake field behavior) may occur. This is

\footnotetext{
*E-Mail: andreas.kabel@desy.de

${ }^{1}$ Zhang Min, private communication
}

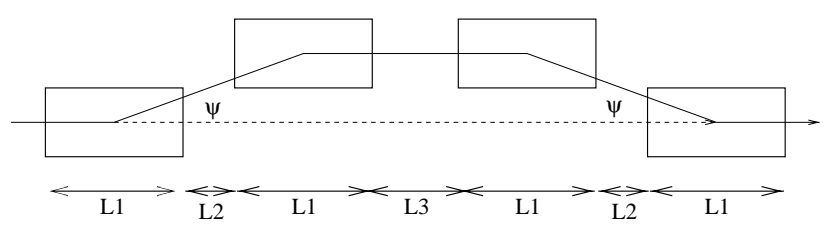

Figure 1: Geometry of BC II: $L_{1}=L_{2}=0.5 \mathrm{~m}, L_{3}=$ $1.3 \mathrm{~m}, \psi=17^{\circ} \ldots 21^{\circ}$

expected to be the main cause of emittance dilution in the TTF bunch compression sections $[3,4,5,6]$.

\section{SLICE EMITTANCE GROWTH}

$\mathrm{TraFiC}^{4}$ is a code written by the authors which allows the numerical computation of these emittance-diluting mechanisms. It is a full three-dimensional tracking code which calculates the fields acting on the particles from first principles. It allows for the calculation of the slice emittance, i. e., the emittance of a longitudinally small sub-ensemble of the bunch. In the FEL process, only particles from within a certain slippage length interact. The saturation length of the process will be affected by the transverse emittance of the sub-ensemble within that slice, while the overall (or projective) emittance only affects beam optics and the brightness of the FEL photon beam. Thus, the slice is the primary quantity to use to judge beam quality. The TTF FEL requires a slice emittance of $\epsilon_{N}<2 \cdot 10^{-6} \mathrm{~m}$. (In this paper, we use the normalized statistical slice emittance $\epsilon_{N}$ with a slice length of $10 \mu \mathrm{m}$ and its counterpart $\epsilon_{N}^{\beta}$ (which is conserved even in dispersive regions for $\delta^{\prime}=0$ ), where $\left(x, x^{\prime}\right)^{\beta}=\left(x, x^{\prime}\right)-\delta\left(\eta, \eta^{\prime}\right)$. ( $x$ refers to the bending plane of motion.)

When one considers a true slice, i. e. an ensemble whose correlation matrix projects to the, $x, x^{\prime}$ subspace, a linear transformation would not generate a dependence of emittance growth on initial Twiss parameters.

Doing exact tracking calculations, however, one finds quite a different behavior: the slice emittance growth depends sensitively on the initial correlation matrix of the incoming slice. We thus can infer that nonlinear effects are an important source of emittance growth.

This fact can also be concluded from typical phase-space portraits of the chicane, indicating an effective sextupole moment in the transport map.

Consequently, the $\mathrm{TraFiC}^{4}$ code has been augmented to 


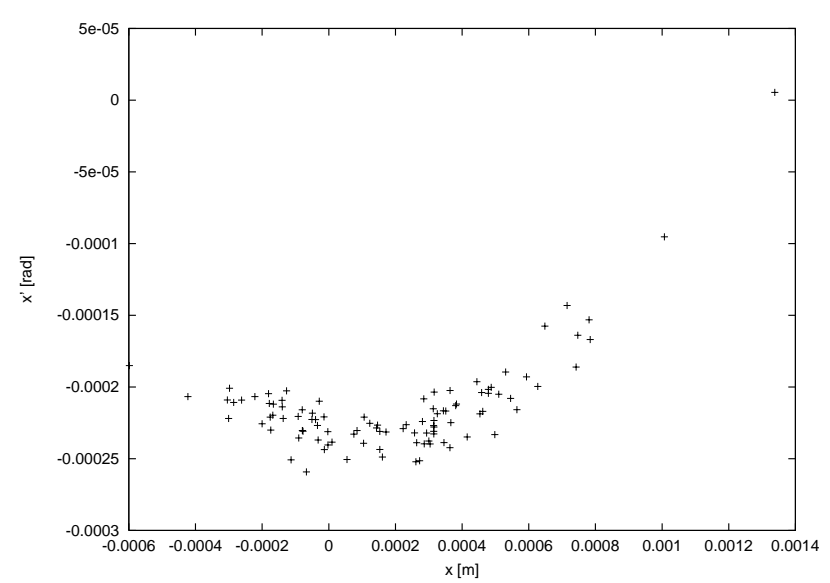

Figure 2: Transverse phase-space portrait of an initially ellipsoidal-gaussian bunch after traveling through the chicane. $\beta_{i}=30 \mathrm{~m}, \epsilon_{N, i}=10^{-6} \mathrm{~m}, \alpha_{i}=0, Q=10^{-9}$ As.

extract nonlinear matrix elements from the transport of the slice through the chicane under the influence of the selffield.

Expanding the transfer map into a power series, one has

$$
\bar{x}_{i}=\sum_{n=0}^{\infty} \sum_{k_{1} \cdots k_{n}=1}^{6} C_{k_{1} \cdots k_{n}}^{(n)} x_{k_{1}} \cdots x_{k_{n}}
$$

where $C^{1}$ is the usual linear transport matrix.

The coefficients $C^{(n)}$ can be read off the transport function through the chicane by tracking an appropriate number of polynomially independent particles and approximating derivatives by difference quotients. For the time being, we are restricted to coefficients up to second order, for which this procedure is quite straightforwardly implemented.

Looking at the dominant coefficients for transverse movement, one finds that, for example, $C_{111}^{2}$ is in the order of magnitude of $10^{5} / \mathrm{m}$, resulting in a significant contribution for realistic beam dimensions.

\subsection{EMITTANCE GROWTH MECHANISMS}

While traveling on the bent trajectory, the test particles will experience collective radiative forces of the bunch as a whole. One has a force longitudinal to the direction of motion, which will induce an energy change, and a transverse force, which will kick the particle of its design orbit. The former causes an angular kick in the dispersive region. Both forces lead to slice emittance growth; in the case of BC II, the growth is clearly dominated by the longitudinal forces, which can be seen by turning off the transverse kicks in the TraFiC ${ }^{4}$ tracking simulations. Thus, the problem is virtually planar, i. e. the motion of the bending plane is decoupled from the one perpendicular to it, in which emittance growth is benign, and we will ignore $\left(y, y^{\prime}\right)$ space in the sequel.

\subsection{OPTICS DEPENDENCE}

We can expect emittance growth due to non-linear dependence of the perturbing fields on the phase space coordinates. In principle, making the bunch extension as small as possible in all directions cures all non-linear effects and we are left with the linear part of emittance growth, so a beam approaching zero-emittance should be left with only the linear effects acting on the emittance.

Starting with a finite-emittance beam, however, the Twiss parameters must be subject to optimization. Making $\left\langle x^{2}\right\rangle$ as small as possible will minimize sampling the nonlinear force component $F_{\|}$components, due to both the reduced size of the sampling slice in the transverse and longitudinal direction.

There are two trade-offs, however: (1) higher $\left\langle x^{2}\right\rangle$ will lead to an induced uncorrelated energy spread, because, to first order, $p_{0} \delta^{\prime}=F_{\|}+x^{\prime} F_{\perp}$ (where the subscripts refer to the design orbit, not the individual particles). Thus, at some point the effects due to the transverse force $F_{\perp}$ will be stronger than the ones due to the variation of $F_{\|}$. One can estimate this break-even point to be at $\beta_{\min } \approx \frac{R\langle F \perp\rangle}{\langle F||\rangle}$, which is well below $10 \mathrm{~cm}$ for BC II. (2) The chicane behaves (in the horizontal plane) basically like a drift space, i.e., a low minimum $\beta$ will lead to a high variation in $\beta$ and thus to uncompensated (see below) sampling of non-linear field components.

The matter is further complicated by the fact that the change in beam dimensions changes not only the sampling range of the test particles, but also the shape of the sampled fields. It turns out, however, that the latter dependence is quite weak as long as the bunch is slim.

The longitudinal fields vary both longitudinally and transversally (for analytic expressions for special cases, cf. [4]). The longitudinal fields scales with the bunch length as $l^{-\frac{4}{3}}$, so one can expect the dominant contribution in the 3rd magnet (where the bunch reaches its final length) and 4th magnet (where the dispersion is closed).

Thus, reduction of the slice emittance growth can be achieved by minimizing the longitudinal dimensions of the bunch as it travels along the chicane. A slice can acquire longitudinal dimension by its initial uncorrelated energy spread and due to its betatron movement. For the transverse slice, where $\eta^{2}\left\langle\delta^{2}\right\rangle \ll\left\langle x^{2}\right\rangle$, the slice length is dominated by the $\beta$ function.

Since in a quadrupole-less magnetic chicane the curvatures of the bending magnets have signature +--+ , the sign of the transverse gradient of the longitudinal field between the magnets changes. A test particle on the concave side of the 3rd bend will be on the convex side of the 4th bend, so in principle the net transverse energy spread will average out.

As the bunch length of the field-generating bunch and its longitudinal distribution changes between magnets, the sampling particles change their relative longitudinal position with respect to the generated field. The field itself will grow in magnitude, as the bunch shortens. Thus, one can- 
not expect this cancellation to be complete.

However, the energy spread can actually decrease within the compressor, which may lead to a decreasing induced angular spread and, consequently, a subsequent lowering of the emittance, which may-in principle-go down to its initial value again. Due to the non-linear components of $F_{\|}$, this mechanism depends on beam dimensions. This mechanism can be observed in figures 2.2,2.2. Similar effects have been exploited in [7].

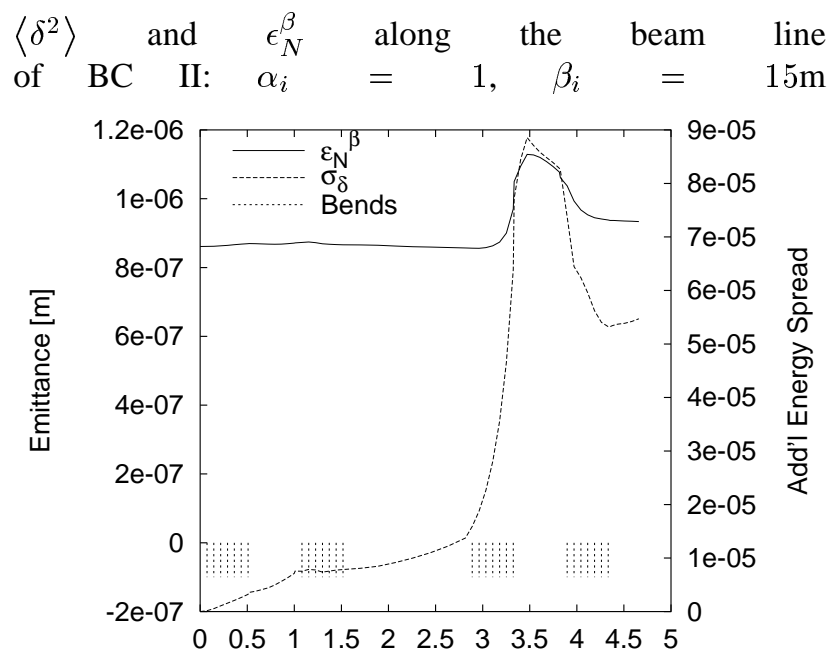

$\left\langle\delta^{2}\right\rangle$ and $\epsilon_{N}^{\beta}$ along the beam line of BC II. $\alpha_{i}=3, \beta_{i}=3 \mathrm{~m}$

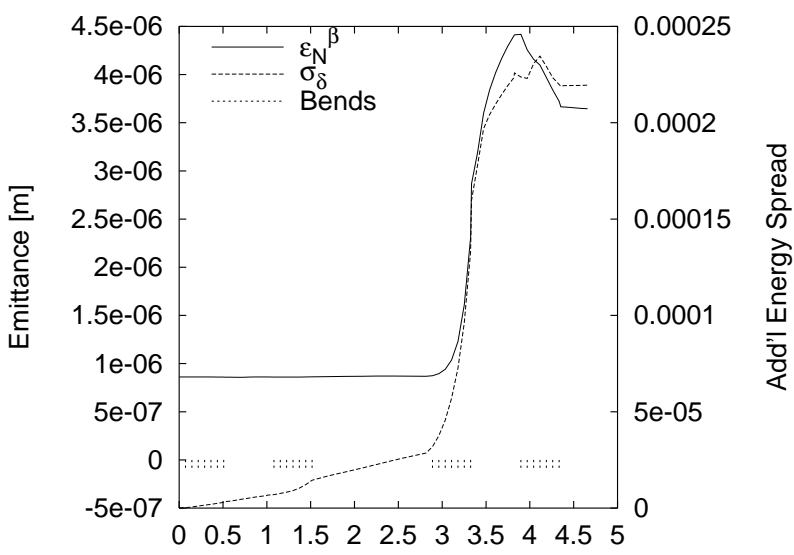

\section{RESULTS}

The $\mathrm{TraFiC}^{4}$ code was used to calculate the emittance growth for a transverse slice of test particles for a range of initial Twiss parameters. The design parameters of $\mathrm{BC}$ II were used. The results are shown in fig. 3. As could be expected from the discussion above, a range of low emittance growth is enclosed by high emittance dilutions both for large and for small $\beta$ functions.

In terms of beam enveloppes, the low $\epsilon$ region corresponds to flat $\left\langle x^{2}\right\rangle(s)$ behavior. The waist of the advantageous $\left\langle x^{2}\right\rangle(s)$ functions is near the 3rd and 4th magnet,
Figure 3: Final emittances for a scan of initial Twiss parameters.

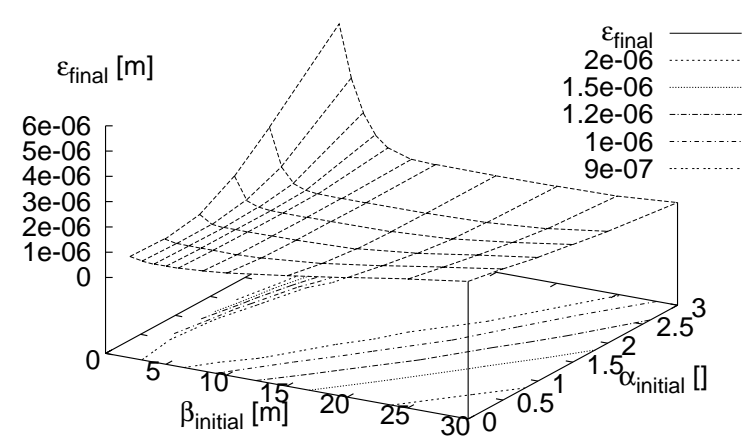

thus causing compensation of energy kicks and minimizing sampling of non-linearities in the region of high field amplitudes.

\section{CONCLUSION}

We have shown that the slice emittance growth in a magnetic chicane can depend heavily on the initial beam parameters chosen, contrary to naïve expectations based on linear estimates. The nonlinear part of the slice emittance growth can be reduced by an appropriate choice of the initial Twiss parameters. For the case of BC II at TTF, the design parameter set should allow a feasible set of optic parameters compatible with the requirements of FEL operation.

\section{REFERENCES}

[1] M. Geitz, A. Kabel, G. Schmidt for the TTF collaboration, "Commissioning of BCII", this conference (unpublished)

[2] D. A. Edwards (Ed.), TESLA Test Facility LINAC - Design Report, DESY Print March 1995, TESLA 95-01

[3] Ya. S. Derbenev, J. Rossbach, E. L. Saldin, V. D. Shiltsev, "Microbunch Radiative Tail - Head Interaction". DESY PRINT-98-023, Sep 1995. 12pp.

[4] Ya. S. Derbenev, V. D. Shiltsev, "Transverse Effects of Microbunch Radiative Interaction”. SLAC-PUB-7181, May 1996.

[5] M. Dohlus, A. Kabel, T. Limberg, "Wake Fields of a Bunch on a General Trajectory Due to Coherent Synchrotron Radiation”, DESY-M-97-10J, August 1997

[6] M. Dohlus, A. Kabel, T. Limberg, "Uncorrelated Emittance Growth in the TTF-FEL Bunch Compression Sections Due to Coherent Synchrotron Radiation and Space Charge Effects", Proceedings of the 6th European Particle Accelerator Conference, 1998

[7] P. Emma, R. Brinkmann "Emittance Dilution Through Coherent Energy Spread Generation in Bending Systems", SLACPUB-7554, May 1997 